\title{
Structural-Property-Relationship in a Series of (Znx4)-Based Metal-Organic Materials
}

\author{
Dinesh Jasrotia* \\ Department of Physics, Govt. Gandhi Memorial Science College, India
}

Submission: June 22, 2017; Published: June 27, 2017

*Corresponding author: Dinesh Jasrotia, Department of Physics, Govt. Gandhi Memorial Science College, Jammu, India, Tel: 01912578189; Fax: 01912578189; Email: phy.dinesh.ap@gmail.com

\begin{abstract}
To scrutinize the role of the weak interactions in structure-property-relationship of zinc based metal-organic materials, a series of compounds were analyzed through single crystal X-ray crystallographic data. The structural parameters were simulated for molecular dynamics to calculate the weak interactions in series of $\mathrm{ZnCl} 2, \mathrm{ZnBr} 2, \mathrm{ZnI} 2$ and $\mathrm{ZnF} 2$ based metal-organic composites. The structural frameworks depict that the metal halides are holding the organic moieties within the inorganic patterns through X-H...A, C-H... $\pi, \pi \ldots \pi$, halogen-halogen and metallophilic weak interactions. The different structural motifs from 1D chain, 2D ribbons and 3D dimmer patterns were observed. The IR and Raman spectra tensors were calculated for the selected series of derivatives which indicates that IR, Raman and Hyper-Raman modes are dominant in $\mathrm{ZnCl} 2$ based derivatives as compared to $\mathrm{ZnBr} 2$, $\mathrm{ZnI} 2$ and $\mathrm{ZnF} 2$ based metal-organic compounds. The structural and spectroscopic parameters reveal that such weak interactions can be used to design the materials with spectroscopic applications.
\end{abstract}

\section{Introduction}

Since, many of the acquitted industrially important materials, such as metals, ceramics or plastics cannot fulfil all the technological desires for the various new applications. Scientists and engineers realized early on that the mixtures of materials can show superior properties as compared with their pure counterparts [1]. It became evident that decreasing the size of the inorganic units to the same level as the organic building blocks could lead to more homogeneous materials that allow a further fine tuning of materials properties on the molecular and structural scale level, generating novel materials that either show characteristics in between the two original phases or even new properties [2]. Inorganic-organic hybrids are classified based on the possible interactions connecting the inorganic and organic species. Class I hybrid materials are those which shows non-covalent interactions between the two phases, such as hydrogen bonding, $\pi . . . \pi$ interactions, halogen...halogen interactions, metallophilic interactions, etc. Class II hybrid materials are those that show strong chemical interactions between the components such as covalent, ionic bonds, etc. [3]. The most obvious advantage of metal-organic hybrids is that they can favourably combine the often dissimilar properties of organic and inorganic components in one material and because of the many possible combinations of components this field is very creative, since it provides the opportunity to invent an almost unlimited set of new materials with a large spectrum of known and unknown properties. While most of these devices can also be produced as fully organic polymer-based systems, the composites with inorganic moieties have important advantages such as the improvement of long term stability, the improvement of electronic properties by doping with functionalized particles and the tailoring of the band gap by changing the size of the particles [3].

\section{Experimental Data}

Motivated by the industrial applications of metal-organic materials, as they are the future of nanotechnology of materials science due to combination of two different branches of solid state sciences such as organic and inorganic, in which the properties of two different materials can be clubbed together into single composite, a series of seventy number of zinc based metal-organic molecules were selected from international union of crystallography, U.K. to analyze the role of secondary interactions in the structure-stability and structure-property relationship. The crystallographic open data base (COD), of international union of crystallography, U.K. was used to gather the available zinc based metal-organic molecules structural data in crystallographic information file format. The molecular dynamics simulations for studying the physical evolutions of weak interactions were performed with DIAMOND-Crystal and molecular structure visualization and function program [4]. 


\section{Results and Discussion}

The hydrogen bond geometry for $\mathrm{ZnCl} 2$ derivatives indicates that the acceptor bond length lies in the range of $1.78 \AA$ to $2.97 \AA$ and the donar-acceptor length exist in between $2.616(2) \AA$ to $3.910(3) \AA ̊$ and $\mathrm{H}$-centered D-A angle has values from $101{ }^{\circ} \mathrm{C}$ to $177^{\circ} \mathrm{C}$.

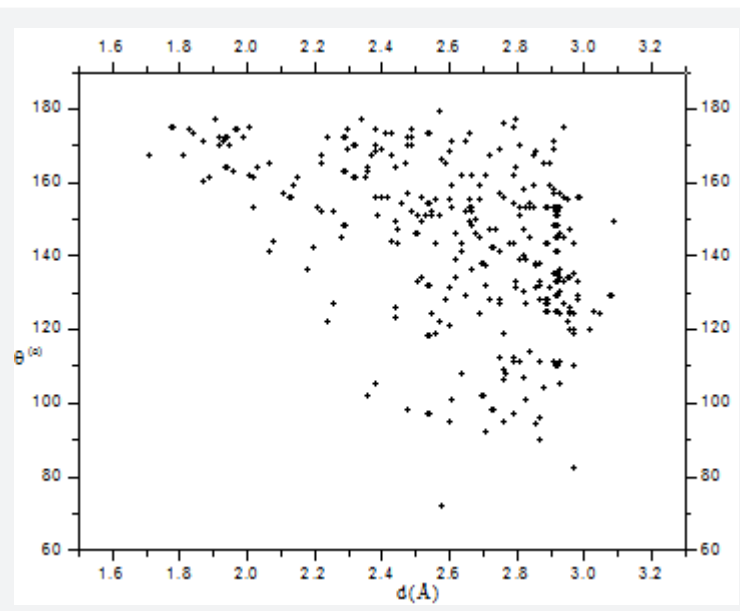

Figure 1: $d(H . . A)$ and $\Theta(X-H . . . A)$ scatter plot for zinc based metal-organic derivatives.

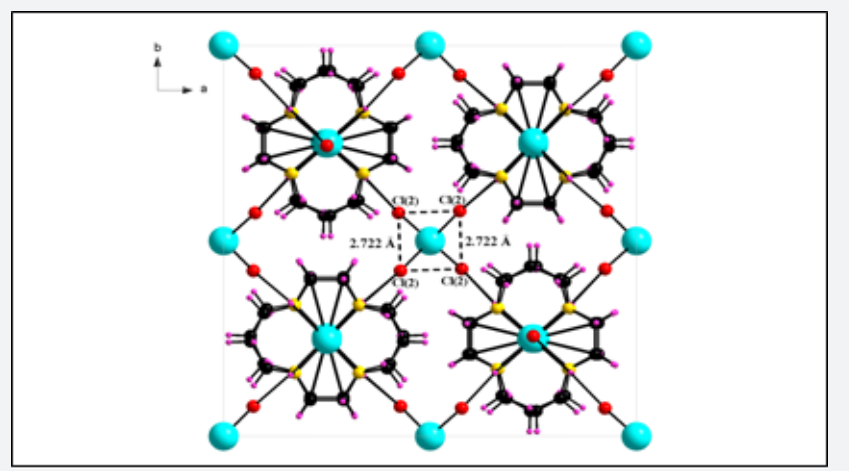

Figure 2: Tetramer pattern of metal-organic $\mathrm{ZnCl1}$ derivative along ab-plane with $\mathrm{Cl}$... Cl $(2.722 \AA)$ interaction at symmetry position 1-y, x, 1-z and 1-x, 1-y, z.

The hydrogen bond geometry for $\mathrm{ZnBr} 2$ derivatives indicates that the acceptor bond length lies in the range of $1.84 \AA$ to $3.08 \AA$ and the donar-acceptor length exist in between $2.732(7) \AA$ to 3.915 (7) and H-centered D-A angle has values from $82{ }^{\circ} \mathrm{C}$ to 179 ${ }^{\circ} \mathrm{C}$. The hydrogen bond geometry for ZnI2 derivatives indicates that the acceptor bond length lies in the range of $2.22 \AA$ to $3.09 \AA$ and the donar-acceptor length exist in between $2.774(9) \AA$ to $3.904(9) \AA$ and $\mathrm{H}$-centered D-A angle has values from $72{ }^{\circ} \mathrm{C}$ to 167 ${ }^{\circ} \mathrm{C}$. The hydrogen bond geometry for $\mathrm{ZnF}_{2}$ derivatives indicates that the acceptor bond length lies in the range of $1.71 \AA$ to $2.92 \AA$ and the donar-acceptor length exist in between $2.677(7) \AA$ to $3.771(6) \AA ̊$ and $\mathrm{H}$-centered D-A angle has values from $94{ }^{\circ} \mathrm{C}$ to $173^{\circ} \mathrm{C}$. The d- $\theta$ scatter plot (Figure $1 \& 2$ ) for hydrogen bonding interactions shows that most of the data points exist in $\mathrm{H}$...A distance range of 2.2 to $2.9 \AA$ and X-H...A angle ranges from 120 ${ }^{\circ} \mathrm{C}$ to $170{ }^{\circ} \mathrm{C}$ in the selected series of metal-organic compounds which indicate these interactions are moderate type of hydrogen bonds [5].

IR and Raman spectra tensors have been calculated by using fractional co-ordinates obtained from single crystal XRD data. In which the lattice parameters and the fractional coordinates were used to study spectral active modes of IR and Raman spectra tensors in the SAM structural utility tool of Bilbao crystallographic server [6,7]. The quantitative comparison of the crystal structures of the selected series of metal-organic compounds through theoretically structural models shows that these compounds have the promising results for spectroscopic applications (Table 1)

Table 1: Hydrogen-Bonding Geometry $(\AA)$ in $\mathrm{ZnCl} 2, \mathrm{ZnBr} 2, \mathrm{ZnI} 2$ and $\mathrm{ZnF} 2$ based derivatives.

\begin{tabular}{|c|c|c|c|c|}
\hline Code & $\mathrm{D}-\mathrm{H}(\mathbf{A})$ & H...A (A) & D...A(A) & $D-H \ldots A(\AA)$ \\
\hline $\mathrm{ZnCl}$ & 0.90 & 2.37 & 3.247 & 139.0 \\
\hline $\mathrm{ZnBr}$ & 0.95 & 2.46 & 3.297 & 130.5 \\
\hline $\mathrm{Znl}$ & 0.86 & 2.65 & 3.339 & 119.5 \\
\hline $\mathrm{ZnF}$ & 0.90 & 2.28 & 3.213 & 133.5 \\
\hline
\end{tabular}

\section{Conclusion}

The analysis of weak interactions such as X-H...A, C-H... $\pi$, $\pi \ldots \pi$, halogen...halogen and $\mathrm{Zn} . . . \mathrm{Zn}$ in zinc based metal-organic derivatives results that these interactions are responsible for holding the inorganic and organic moieties in single composite. The $\pi \ldots \pi$ interactions are binding the organic moieties into dimmer patterns whereas the halogen-halogen and metallophilic interactions are holding the inorganic constituents into 1D chain and 2D layer patterns. The metallic constituents of these derivatives provide the mechanical strength to the composite whereas the organic moiety is responsible for its optical as well as spectroscopic properties. The spectroscopic studies for IR, Raman and Hyper-Raman tensors show that these materials can be used in junction diodes of hybrid solar cells in which the stability of the hybrid structure is maintained by these weak interactions. Looking upon the industrial applications of hybrid material, they are the future of nanotechnology due to combination of two different branches of solid state sciences such as organic and inorganic in which the properties of two different scientific worlds were clubbed together into single composite material.

\section{Aknowledgement}

The corresponding author (Dinesh Jasrotia) is thankful to University Grants Commission (UGC) for research funding under UGC-Major Research Project no. 42-777 of 2013. 


\section{References}

1. Kazemipoor M, Hamzah S, Hajifaraji M, Radzi CW, Cordell GA (2016) Slimming and Appetite-Suppressing Effects of Caraway Aqueous Extract as a Natural Therapy in Physically Active Women. Phytother Res 30(6): 981-987.

2. Pourahmadi M, Jahromi H, Rooeintan Y (2016) The effect of aqueous extract of caraway seed (Carum carvi) on Cholecystokinin hormone in male rat. Journal of Fundamental and Applied Sciences 8(4): 20232035.

3. Kazemipoor M, Hajifaraji M, Haerian BS, Mosaddegh MH, Cordell GA, et al. (2013) Antiobesity effect of caraway extract on overweight and obese women: a randomized, triple-blind, placebo-controlled clinical trial. Evidence-Based Complementary and Alternative Medicine, pp. 8.

4. Saghir MR, Sadiq S, Nayak S, Tahir MU (2012) Hypolipidemic effect of aqueous extract of Carum carvi (black Zeera) seeds in diet induced hyperlipidemic rats. Pak J Pharm Sci 25(2): 333-337.

5. Jouad H, Haloui M, Rhiouani H, El Hilaly J, Eddouks M (2001) Ethnobotanical survey of medicinal plants used for the treatment of diabetes, cardiac and renal diseases in the North centre region of Morocco (Fez-Boulemane). J Ethnopharmacol 77(2-3):175-182.
6. Agrahari P, Singh DK (2014) A review on the pharmacological aspects of Carum carvi. Journal of Biology and earth sciences 4(1):1-13.

7. Haidari F, Seyed-Sadjadi N, Taha-Jalali M, Mohammed-Shahi M (2011) the effect of oral administration of Carum carvi on weight, serum glucose, and lipid profile in streptozotocin-induced diabetic rats. Saudi Med J 32(7): 695-700.

8. Kazemipoor M, Radzi CW, Hajifaraji M, Cordell GA (2014) Preliminary Safety Evaluation and Biochemical Efficacy of a Carum carvi Extract: Results from a Randomized, Triple-Blind, and Placebo-Controlled Clinical Trial. Phytother Res 28(10): 1456-1460.

9. Samojlik I, Lakić N, Mimica-Dukić N, Đaković-Švajcer K, Božin B (2010) Antioxidant and hepatoprotective potential of essential oils of coriander (Coriandrum sativum L.) and caraway (Carum carvi L.) (Apiaceae). J Agric Food Chem 58(15): 8848-8853.

10. Kamaleeswari M, Nalini N (2006) Dose-response efficacy of caraway (Carum carvi L.) on tissue lipid per oxidation and antioxidant profile in rat colon carcinogenesis. Journal of pharmacy and pharmacology 58(8):1121-1130.

11. Lemhadri A, Hajji L, Michel J-B, Eddouks M (2006) Cholesterol and triglycerides lowering activities of caraway fruits in normal and streptozotocin diabetic rats. J Ethnopharmacol 106(3): 321-326.

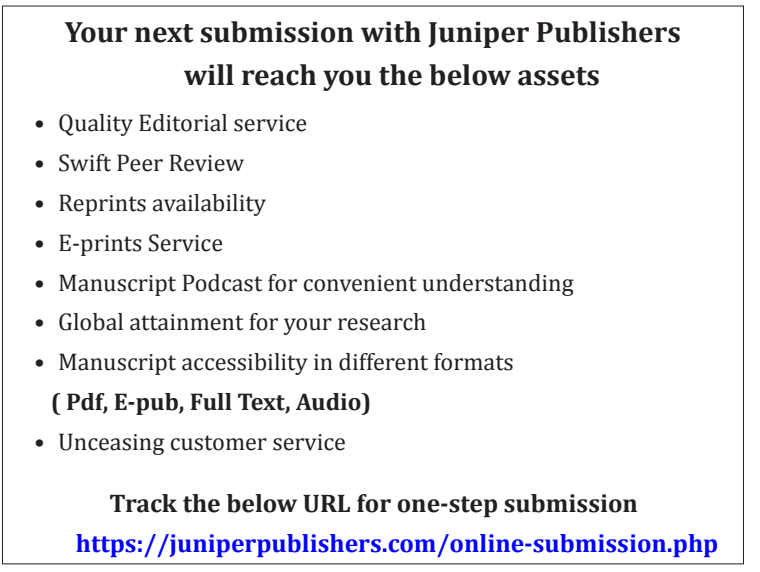

